\title{
On the Design of Economic NMPC based on Approximate Turnpike Properties
}

\author{
Timm Faulwasser $^{a, b}$ and Dominique Bonvin ${ }^{a}$
}

\begin{abstract}
We discuss the design of sampled-data economic nonlinear model predictive control schemes for continuous-time systems based on turnpike properties. In a recent paper we have shown that an exact turnpike property allows establishing finitetime convergence of the NMPC scheme to the optimal steady state, and also recursive feasibility, without using terminal penalties or terminal constraints. Herein, we extend our previous results to the more general case of approximate turnpikes. We establish sufficient conditions, based on a dissipativity assumption, that guarantee (i) convergence to a neighborhood of the optimal steady state, and (ii) recursive feasibility in the presence of state constraints. The proposed conditions do not rely on terminal regions or terminal penalties. A key step in our developments is the use of a storage function as a penalty on the initial condition in the NMPC scheme. We draw upon the example of a chemical reactor to illustrate our findings.

Index Terms-dissipativity, turnpike property, economic model predictive control, nonlinear model predictive control, convergence
\end{abstract}

\section{INTRODUCTION}

Recently, there has been a widespread interest in nonlinear model predictive control (NMPC) schemes that are tailored to optimizing transient performance instead of stabilizing a system at a set point. The name economic MPC is used in the literature for these approaches [5]. It is well understood that turnpike properties are an intrinsic feature of optimal control problems (OCP) arising in economic NMPC $[5,6,10]$. The term turnpike describes a property of OCPs, whereby, for varying initial condition and horizon, the optimal solutions stay close to a specific steady state during the major part of the time horizon $[1,7,12]$. However, only very few papers use turnpike properties explicitly to establish convergence and/or stability conditions for economic NMPC $[6,10,11]$.

In [10], the stability of economic NMPC without terminal constraint or end penalties is proven based on a dissipativity assumption. Essentially, the dissipativity condition is used to establish turnpike behavior. The stability proof of [10], however, assumes feasibility of the underlying optimization at each sampling instant. Similarly, the construction of a Lyapunov function for economic NMPC without terminal constraints presented in [11] assumes recursive feasibility in the presence of state constraints. ${ }^{1}$

\footnotetext{
${ }^{a} \mathrm{TF}$ and $\mathrm{DB}$ are with the Laboratoire d'Automatique, École Polytechnique Fédérale de Lausanne, CH-1015 Lausanne, Switzerland. \{timm.faulwasser, dominique.bonvin\}@epfl.ch

${ }^{b} \mathrm{TF}$ is also with the Institute for Applied Computer Science, Karlsruhe Institute of Technology, 76131 Karlsruhe, Germany.

${ }^{1}$ More precisely, it is assumed in $[10,11]$ that the OCP admits infinitehorizon admissible solutions for any initial condition, which directly implies that the state constraints are a control-invariant set.
}

In [6], we have shown in a sampled-data continuous-time setting that exact turnpike properties, which require the openloop optimal solutions to be at the optimal steady state during a subinterval of the optimization horizon, can be used to establish both finite-time convergence to the optimal steady state and recursive feasibility. The results in [6] do not rely on terminal penalties or terminal constraints. The contribution of the present paper extends [6] to the more general case of approximate turnpikes based on a dissipativity assumption. ${ }^{2}$ A key step in our developments is the use of a storage function as a penalty on the initial condition in the NMPC scheme. Based on this, we present conditions guaranteeing the convergence to a neighborhood of the optimal steady state. In contrast to $[10,11]$, we work in a sampled-data continuous-time setting, and we do not assume recursive feasibility. Instead, we rely on local controllability in a neighborhood of the turnpike to establish recursive feasibility in the presence of state constraints.

The remainder of the paper is structured as follows. Section II describes a general sampled-data NMPC scheme. The notion of turnpike properties of OCPs and the implications of turnpike properties are discussed in Section III. The main NMPC stability result is presented in Section IV. We draw upon a chemical reactor example to illustrate our findings in Section V.

\section{SAMPLED-DATA NMPC}

We consider the nonlinear input-affine plant given by

$$
\dot{x}_{p}=f\left(x_{p}\right)+\sum_{i=1}^{n_{u}} g_{i}\left(x_{p}\right) u_{p, i}, \quad x_{p}(0)=x_{0} \in \mathcal{X}_{0},
$$

where the state $x_{p} \in \mathbb{R}^{n_{x}}$ and the input $u_{p} \in \mathbb{R}^{n_{u}}$ are constrained to the compact sets $\mathcal{X} \subset \mathbb{R}^{n_{x}}$ and $\mathcal{U} \subset \mathbb{R}^{n_{u}}$. The initial condition $x_{0}$ is constrained to the compact set $\mathcal{X}_{0} \subseteq \mathcal{X}$. We assume that $f: \mathbb{R}^{n_{x}} \rightarrow \mathbb{R}^{n_{x}}$ and $g_{i}: \mathbb{R}^{n_{x}} \rightarrow$ $\mathbb{R}^{n_{x}}$ are Lipschitz on $\mathcal{X}$ and sufficiently often continuously differentiable.

We are interested in controlling the plant (1) by means of a sampled-data NMPC scheme. The NMPC scheme is based on receding-horizon solutions to an OCP. Hence, at each sampling instant $t_{k}=k \delta, k \in \mathbb{N}$, we propose to minimize the objective functional

$$
J\left(x_{p}(0), u(\cdot)\right):=I\left(x_{p}(0)\right)+\int_{0}^{T} F(x(\tau), u(\tau)) d \tau,
$$

\footnotetext{
${ }^{2}$ We use the term approximate turnpike to highlight that the open-loop optimal solutions do not need to reach the turnpike steady state exactly. It suffices if they are, for some time, in any arbitrarily small neighborhood of it, cf. [6].
} 
where $F: \mathcal{X} \times \mathcal{U} \rightarrow \mathbb{R}_{0}^{+}$is the Lipschitz continuous cost function, $T \in(0, \infty)$ is the prediction horizon, and $\delta>0$ is the sampling time. In contrast to standard formulations of NMPC, we consider a penalty of the initial condition $I\left(x_{p}\left(t_{k}\right)\right)$, the purpose of which will be discussed later. Furthermore, we distinguish between the plant variables in (1) and the values predicted by the model by denoting the former with the subscript $(\cdot)_{p}$ and the latter without subscript. The NMPC scheme is based on receding-horizon solutions to the following OCP

$$
\underset{u(\cdot) \in \mathcal{L}([0, T], \mathcal{U})}{\operatorname{minimize}} J\left(x_{p}\left(t_{k}\right), u(\cdot)\right)
$$

subject to, for all $\tau \in[0, T]$,

$$
\begin{aligned}
\frac{d x(\tau)}{d \tau} & =f(x(\tau))+\sum_{i=1}^{n_{u}} g_{i}(x(\tau)) u_{i}(\tau) \\
x(0) & =x_{p}\left(t_{k}\right) \\
u(\tau) & \in \mathcal{U}, x(\tau) \in \mathcal{X}
\end{aligned}
$$

where $\mathcal{L}([0, T], \mathcal{U})$ denotes the class of measurable functions on $[0, T]$ taking values in the compact set $\mathcal{U}$.

The purpose of the subsequent developments is to establish conditions ensuring that NMPC based on (3) leads to asymptotic convergence to a neighborhood of a specific steady state. To this end, we make the following assumptions.

Assumption 1: Model (3b) is identical to the plant (1), i.e., there is no plant-model mismatch.

Assumption 2: For any $x_{0} \in \mathcal{X}$ and any input $u(\cdot) \in$ $\mathcal{L}([0, \infty), \mathcal{U})$, plant $(1)$ has a unique absolutely continuous solution.

Let $x\left(\cdot, x_{p}\left(t_{k}\right), u(\cdot)\right)$ denote a solution to (3b) that starts at $x_{p}\left(t_{k}\right)$ at time $\tau=0$ and is driven by the input $u(\cdot) \in \mathcal{L}([0, T]), \mathcal{U})$. An optimal solution to (3) is denoted as $u^{\star}\left(\cdot, x_{p}\left(t_{k}\right)\right)$, the corresponding state trajectory is $x^{\star}\left(\cdot, x_{p}\left(t_{k}\right)\right):=x^{\star}\left(\cdot, x_{p}\left(t_{k}\right), u^{\star}\left(\cdot, x_{p}\left(t_{k}\right)\right)\right)$, and the corresponding optimal value of (2) is

$$
J^{\star}\left(x_{p}\left(t_{k}\right)\right):=J\left(x_{p}\left(t_{k}\right), u^{\star}\left(\cdot, x_{p}\left(t_{k}\right)\right)\right) .
$$

At the sampling instant $t_{k}$, the first part of the optimal solution $u^{\star}\left(\cdot, x_{p}\left(t_{k}\right)\right)$ is applied to (1), i.e.

$$
u_{p}\left(t_{k}+\tau\right)=u^{\star}\left(\tau, x_{p}\left(t_{k}\right)\right), \quad \forall \tau \in[0, \delta) .
$$

Notational remarks. We denote the dependence of (3) on the initial condition $x_{p}\left(t_{k}\right)$ and the horizon length $T$ arising from the receding-horizon control strategy by writing $\mathcal{O C P}_{T}\left(x_{p}\left(t_{k}\right)\right)$. While the time variable of the plant (1) is $t \geq 0$, the time variable of $\mathcal{O C P}_{T}\left(x_{p}\left(t_{k}\right)\right)$ is denoted by $\tau \in[0, T]$. The pair $z(\cdot):=\left(x\left(\cdot, x_{p}\left(t_{k}\right), u(\cdot)\right), u(\cdot)\right)^{T}$ is said to be admissible if $u(\cdot) \in \mathcal{L}([0, T], \mathcal{U})$ and, for all $\tau \in[0, T], x\left(\tau, x_{p}\left(t_{k}\right), u(\cdot)\right) \in \mathcal{X}$. Occasionally, we want to highlight the dependence of an admissible pair $z(\cdot)$ on the initial condition $x_{p}\left(t_{k}\right)$, for which we write $z\left(\cdot, x_{p}\left(t_{k}\right)\right)$ and

$$
F\left(z\left(\tau, x_{p}\left(t_{k}\right)\right)\right):=F\left(x\left(\tau, x_{p}\left(t_{k}\right), u(\cdot)\right), u(\tau)\right) .
$$

Steady-state values are indicated by the superscript $\left(^{(}\right)$, and thus we denote steady-state pairs by $\bar{z}:=(\bar{x}, \bar{u})^{T}$. The combined input and state constraints are written as $\mathcal{Z}:=$ $\mathcal{X} \times \mathcal{U}$.

Remark 1 (Role of $I\left(x_{p}\left(t_{k}\right)\right)$ in $\left.\mathcal{O C P}_{T}\left(x_{p}\left(t_{k}\right)\right)\right)$ :

Note that, whenever $x_{p}\left(t_{k}\right)$ is not a decision variable of the OCP, which will be the case in our developments, the penalty on the initial condition, $I$ in (2), is a mere translation of $J$. Consequently, and without loss of generality, we analyze in Sections III-B and III-C the questions of feasibility and existence of turnpikes without specifying $I$ explicitly. In order to establish decrease of $J^{\star}\left(x_{p}\left(t_{k+1}\right)\right)-J^{\star}\left(x_{p}\left(t_{k}\right)\right)$ in Section III-D, we will consider $I\left(x_{p}\left(t_{k}\right)\right) \neq 0$.

\section{TURNPIKE PROPERTIES OF OCPS}

This paper investigates sufficient conditions for the convergence of plant (1) subject to the sampled-data NMPC scheme (3). These conditions rely on turnpike properties that describe features of solutions to an OCP for varying initial condition. To this end, we consider in this section $\mathcal{O C P} \mathcal{P}_{T}\left(x_{0}\right)$ with $x_{0} \in \mathcal{X}_{0}$, i.e., we consider solutions to (3) for varying initial condition.

\section{A. Input-state turnpike}

Definition 1 (Input-state turnpike property [6, 7]):

The optimal solution pairs $z^{\star}\left(\cdot, x_{0}\right)$ of $\mathcal{O C} \mathcal{P}_{T}\left(x_{0}\right)$ are said to have an input-state turnpike property with respect to the steady-state pair $\bar{z}=(\bar{x}, \bar{u})^{T} \in \mathcal{Z}$ if there exists a function $\nu:[0, \infty) \rightarrow[0, \infty]$ such that, for all $x_{0} \in \mathcal{X}_{0}$ and all $T>0$, we have

$$
\mu\left[\Theta_{\varepsilon, T}\right]<\nu(\varepsilon)<\infty \quad \forall \varepsilon>0,
$$

where $\mu[\cdot]$ is the Lebesgue measure on the real line and

$$
\Theta_{\varepsilon, T}:=\left\{\tau \in[0, T]:\left\|z^{\star}\left(\tau, x_{0}\right)-\bar{z}\right\|>\varepsilon\right\} .
$$

The pairs $z^{\star}\left(\cdot, x_{0}\right)$ of (3) are said to have an exact input-state turnpike property if Condition (6) also holds at $\varepsilon=0$, i.e.,

$$
\mu\left[\Theta_{0, T}\right]<\nu(0)<\infty .
$$

The term turnpike property has been coined by [4]. It states that-for any initial condition $x_{0} \in \mathcal{X}_{0}$ and any horizon length $T>0$ - the time that the optimal pairs spend outside an $\varepsilon$-neighborhood of $\bar{z}$ is bounded by $\nu(\varepsilon)$, where $\nu(\varepsilon)$ is not a function of the horizon length $T$. In essence, the turnpike property states the existence of an arc along which the optimal pair $z^{\star}(\cdot)$ stays close to the steady-state pair $\bar{z}$ in the sense of the Euclidean norm $\|\cdot\|$, and the time length of this arc increases with increasing horizon length $T$.

Definition 1 is a variant of that used in [7], where turnpike solutions are required to be close only to the steady state $\bar{x}$, which may be denoted as a state turnpike. In contrast, we require here that the turnpike solutions are close to the steady-state values of the state and the input, which we denote as a input-state turnpike. A definition of the turnpike property based on exponential bounds on the trajectories is used in [2] for discrete-time problems. For continuous-time systems, a definition similar to (6) is implicitly given in [1]. 


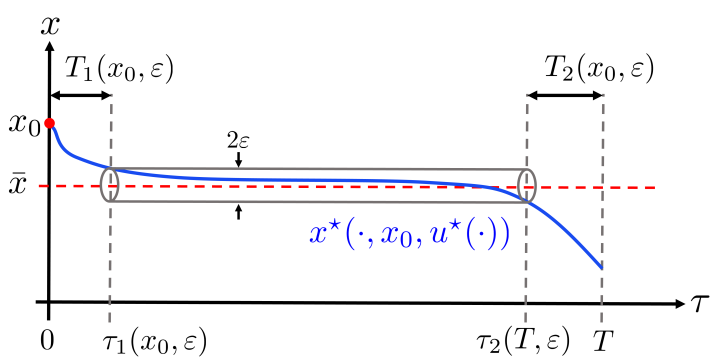

Fig. 1. Sketch of an approximate turnpike property.

It is fair to ask whether there are conditions ensuring the existence of turnpike properties. The following result is given, in slightly different form, in [7].

Proposition 1 (Existence of turnpikes [7]):

Let the data of $\mathcal{O C P}_{T}\left(x_{0}\right)$ satisfy:

(i) There exists a bounded function $S: \mathcal{X} \rightarrow \mathbb{R}_{0}^{+}$, such that, for all $x_{0} \in \mathcal{X}_{0}$, some class- $\mathcal{K}$ function $\alpha$, and any admissible pair $z\left(\cdot, x_{0}\right)$, with $z\left(t, x_{0}\right) \in \mathcal{Z}$ for all $t \in[0, T]$, and $x_{T}=x\left(T, x_{0}, u(\cdot)\right) \in \mathcal{X}$, the inequality

$$
\begin{aligned}
S\left(x_{T}\right)-S\left(x_{0}\right) \leq \int_{0}^{T} & -\alpha\left(\left\|z\left(\tau, x_{0}\right)-\bar{z}\right\|\right) \\
& +F\left(z\left(\tau, x_{0}\right)\right)-F(\bar{z}) d \tau
\end{aligned}
$$

holds.

(ii) For all $x_{0} \in \mathcal{X}_{0}$, the steady state $\bar{x}$ is reachable, without violation of input or state constraints, in some time $\hat{T}(\bar{x})<\infty$.

Then, for all $x_{0} \in \mathcal{X}_{0}, \mathcal{O C P}_{T}\left(x_{0}\right)$ has an input-state turnpike at $\bar{z}$.

This result is a minor extension to Theorem 1 in [7]. Therein, (9) with $\alpha(\|x-\bar{x}\|)$ is used to establish a state turnpike at $\bar{x}$. Here, (9) with $\alpha(\|z-\bar{z}\|)$ is used to establish an inputstate turnpike at $\bar{z}$. Additionally, we require here for technical reasons that the storage function $S$ be positive on $\mathcal{X}$. Due to space limitations and the fact that one can easily adapt the proof given in [7], we omit a detailed proof.

It is worth mentioning that (9) is a strict dissipation inequality, whereby $F(z)-F(\bar{z})$ can be understood as a supply rate and $S$ is a storage function. Similar conditions are used frequently in the context of economic NMPC [3, 10]. Note, however, that $\mathcal{X}_{0} \neq \mathcal{X}$, and hence Condition (i) of Proposition 1 is slightly more general than a dissipativity assumption. We also note that (9) implies that the steadystate minimum of $F$ on $\mathcal{Z}$ is attained at $\bar{z}$. In other words, $\bar{z}$ is the unique optimal steady-state pair, cf. [7, Cor. 1]

In principle, the measure-based approach of Definition 1 allows for pathological cases in which a pair $z^{\star}\left(\cdot, x_{0}\right)$ intersects a small neighborhood of the turnpike infinitely often during a compact interval of time. To avoid such cases we make the following assumption. Let $\overline{\mathcal{B}}_{\varepsilon}(\bar{z})$ be a closed ball of radius $\varepsilon$ centered at $\bar{z}$.

Assumption 3 (Non-pathological turnpike): (i) $\mathcal{O C P}_{T}\left(x_{0}\right)$ is such that Conditions (i)-(ii) of Proposition 1 are satisfied for all $x_{0} \in \mathcal{X}_{0}$, i.e., for all $x_{0} \in \mathcal{X}_{0}$, $\mathcal{O C P}_{T}\left(x_{0}\right)$ has an input-state turnpike at $\bar{z}$.

(ii) If $\mathcal{O C P}_{T}\left(x_{0}\right)$ has a turnpike, then it is non-pathological, i.e., there exists $\hat{\varepsilon}>0$ such that for all $\varepsilon \in(0, \hat{\varepsilon}]$ and sufficiently large $T>0$, we can find nonnegative constants $T_{1}\left(x_{0}, \varepsilon\right)$ and $T_{2}\left(x_{0}, \varepsilon\right)$ such that $T_{1}\left(x_{0}, \varepsilon\right) \leq T-T_{2}\left(x_{0}, \varepsilon\right)$. Upon defining $\tau_{1}\left(x_{0}, \varepsilon\right):=$ $T_{1}\left(x_{0}, \varepsilon\right)$ and $\tau_{2}(T, \varepsilon):=T-T_{2}\left(x_{0}, \varepsilon\right),{ }^{3}$ the optimal pairs $z^{\star}\left(\cdot, x_{0}\right)$ satisfy $z^{\star}\left(\tau, x_{0}\right) \in \overline{\mathcal{B}}_{\varepsilon}(\bar{z})$ for all $\tau \in$ $\left[\tau_{1}\left(x_{0}, \varepsilon\right), \tau_{2}(T, \varepsilon)\right]$.

The main idea of part (ii) of this assumption is sketched in Figure 1. In essence, we assume that the solutions to $\mathcal{O C P}_{T}\left(x_{0}\right)$ are such that each sufficiently small $\varepsilon$ neighborhood of the turnpike $\bar{z}$ is entered at time $\tau_{1}\left(x_{0}, \varepsilon\right)$. The solutions stay within the $\varepsilon$-neighborhood during the interval $\left[\tau_{1}\left(x_{0}, \varepsilon\right), \tau_{2}(T, \varepsilon)\right]$ and leave the $\varepsilon$-neighborhood of $\bar{z}$ at $\tau_{2}(T, \varepsilon)$.

\section{B. Turnpikes in sequences of $\mathcal{O C P}_{T}\left(x_{0}\right)$}

We are interested in establishing conditions under which the existence of a turnpike in $\mathcal{O C P}_{T}\left(x_{0}\right)$ implies the existence of the same turnpike in the sequence of OCPs arising in the context of NMPC. If the conditions of Proposition 1 are satisfied for $\mathcal{X}_{0}=\mathcal{X}$, then $\mathcal{O C P}_{T}\left(x_{0}\right)$ has a turnpike at $\bar{z}$ for any $x_{0} \in \mathcal{X}$. This, in turn, would require the set of feasible states $\mathcal{X}$ to be a control-invariant set, which is in general a very strong assumption. Hence, we derive sufficient conditions for the case $\mathcal{X}_{0} \subset \mathcal{X}$ ensuring that the sequence of OCPs arising in the context of NMPC has the turnpike property if $\mathcal{O C P} \mathcal{P}_{T}\left(x_{0}\right)$ has it.

To this end, we introduce a controllability assumption and recall results on small-time local controllability. Let int $\mathcal{Z}$ denote the interior of the set $\mathcal{Z}$.

Assumption 4 (Local controllability at $\bar{z}$ ): The turnpike steady-state pair satisfies $\bar{z} \in \operatorname{int} \mathcal{Z}$, and the Jacobian linearization of (1) at $\bar{z}$ is controllable.

Let $L_{f}$ be the Lipschitz constant of (1) with respect to $(x, u)$, let $\mathcal{B}_{\varepsilon}(\bar{x})$ be an open ball of radius $\varepsilon$ centered at $\bar{x}$, and let $\overline{\mathcal{B}}_{\varepsilon}(\bar{x})$ denote the closure of $\mathcal{B}_{\varepsilon}(\bar{x})$.

Lemma 1 (Small-time local controllability): Let Assumption 4 hold. Then, for any $\delta>0$ and any $\eta>0$, there exist constants $\sigma>\varepsilon>0$ such that the following statements hold:

(i) For all $x_{0} \in \mathcal{B}_{\varepsilon}(\bar{x})$, there exists $u(\cdot) \in$ $\mathcal{L}\left(\left[0, \frac{1}{2} \delta\right], \mathcal{B}_{\eta}(\bar{u})\right.$ such that

$$
x\left(\frac{1}{2} \delta, x_{0}, u(\cdot)\right)=\bar{x} .
$$

(ii) For all $x_{0} \in \mathcal{B}_{\varepsilon}(\bar{x})$, there exists $u(\cdot) \in \mathcal{L}\left([0, \delta], \mathcal{B}_{\eta}(\bar{u})\right)$ such that

$$
x\left(\delta, x_{0}, u(\cdot)\right)=x_{0} .
$$

(iii) For all $\tau \in[0, \delta]$, we have $x\left(\tau, x_{0}, u(\cdot)\right) \in \overline{\mathcal{B}}_{\sigma}(\bar{x})$, with $\sigma=\varepsilon+(\varepsilon+\eta)\left(e^{L_{f} \delta}-1\right)$.

\footnotetext{
${ }^{3}$ Note that, in general, $\tau_{2}(T, \varepsilon)$ depends on $T, \varepsilon$ and $x_{0}$. For the sake of readability, we drop the argument $x_{0}$ and write $\tau_{2}(T, \varepsilon)$.
} 
Proof: The proof of parts (i) and (ii) follows directly from [14, Prop. 11.2, p. 51]. The bound in part (iii) is implied by Lemma 5 given in the Appendix.

Next, we consider

$$
x_{\delta}:=x^{\star}\left(\delta, x_{0}, u^{\star}\left(\cdot, x_{0}\right)\right),
$$

which is the state reached from $x_{0}$ after one sampling time upon application of the optimal input $u^{\star}\left(\cdot, x_{0}\right)$.

Lemma 2 (Turnpikes in sequences of $\mathcal{O C P}_{T}\left(x_{0}\right)$ ): Let the conditions of Proposition 1 be satisfied for $\mathcal{X}_{0}=\left\{x_{0}\right\}$, and let Assumption 4 hold. Then, there exists $\hat{\delta}>0$ such that, for all $\delta \in(0, \hat{\delta})$, the conditions of Proposition 1 are also satisfied for $\mathcal{X}_{0}=\left\{x_{\delta}\right\}$.

Proof: From (10) it follows that $x_{\delta}$ is reachable from from $x_{0}$. Hence, if inequality (9) holds for any admissible pair originating form $x_{0}$, it also holds for any admissible pair originating from $x_{\delta}$. Therefore, Condition (i) of Proposition 1 is satisfied at $x_{\delta}$.

Without loss of generality, the input-state turnpike property of $\mathcal{O C P} \mathcal{P}_{T}\left(x_{0}\right)$ implies that we can always pick a horizon $T<\infty$ long enough such that, for any $\varepsilon>0$, there exists a time $\tilde{\tau}$, with

$$
z^{\star}\left(\tilde{\tau}, x_{0}\right) \in \mathcal{B}_{\varepsilon}(\bar{z}) .
$$

Consider any sampling time $\delta$ with $\delta<\tilde{\tau}$. This implies that, from $x_{\delta}$, one can always reach an arbitrarily small neighborhood $\mathcal{B}_{\varepsilon}(\bar{x})$ of the turnpike state $\bar{x}$ by application of $u^{\star}\left(\tau, x_{0}\right)$ during $\tau \in[\delta, \tilde{\tau}]$.

Now, pick $T$ sufficiently large, which implies that $\varepsilon$ is sufficiently small to apply part (i) of Lemma 1 . Hence, the nonlinear system (1) can be steered, in finite time and by means of an admissible input, from $z^{\star}\left(\tilde{\tau}, x_{0}\right)$ to $\bar{z}$. In the view of (11), it follows that, for all $\delta \in[0, \tilde{\tau}]$, the turnpike $\bar{x}$ is reachable in finite time from $x_{\delta}$. Hence, Condition (ii) of Proposition 1 holds at $x_{\delta}$.

Lemma 2 has an important implication: If, for all $x_{0} \in$ $\mathcal{X}_{0}, \mathcal{O C P} \mathcal{P}_{T}\left(x_{0}\right)$ has a turnpike, then, for sufficiently small sampling times $\delta$ and sufficiently long horizons $T$, it holds that $x_{\delta} \in \mathcal{X}_{0}$. In other words, provided controllability holds at $\bar{z} \in$ int $\mathcal{Z}$, one can choose $T$ and $\delta$ such that all elements of the sequence of $\mathcal{O C P}_{T}\left(x_{p}\left(t_{k}\right)\right)$ that arise in the context of NMPC have a turnpike at $\bar{z}$.

\section{Recursive feasibility of $\mathcal{O C P}_{T}\left(x_{0}\right)$}

Now, we shift the focus to the feasibility properties of sequences of $\mathcal{O C P}_{T}\left(x_{0}\right)$. Recall that Assumption 4 via Lemma 1 implies that, for any sufficiently small $\delta>0$ and any $\eta>0$, there exist $\sigma, \varepsilon$, with $\sigma>\varepsilon>0$, such that for each $x_{\varepsilon} \in \mathcal{B}_{\varepsilon}(\bar{x})$ we can find $u_{\varepsilon}\left(\cdot, x_{\varepsilon}\right) \in \mathcal{L}\left([0, \delta], \mathcal{B}_{\eta}(\bar{u})\right)$ satisfying

$$
\begin{aligned}
\forall \tau \in[0, \delta]: \quad & x\left(\tau, x_{\varepsilon}, u_{\varepsilon}\left(\cdot, x_{\varepsilon}\right)\right) \in \overline{\mathcal{B}}_{\sigma}(\bar{x}) \subset \mathcal{X} \\
& x\left(\delta, x_{\varepsilon}, u_{\varepsilon}\left(\cdot, x_{\varepsilon}\right)\right)=x_{\varepsilon} .
\end{aligned}
$$

In other words, at all states in a sufficiently small neighborhood of $\bar{x}$, one can construct an admissible periodic orbit of length $\delta$. One should note that this periodic trajectory is contained in $\overline{\mathcal{B}}_{\sigma}(\bar{x})$, with $\sigma$ given in part (iii) of Lemma 1 . If the additional condition

$$
\mathcal{B}_{\varepsilon}(\bar{x}) \subset\{x \in \mathcal{X} \mid \exists u \in \mathcal{U}: \quad f(x, u)=0\}
$$

holds, then one can show that $u_{\varepsilon}\left(\tau, x_{\varepsilon}\right)=u\left(x_{\varepsilon}\right)=$ const. In this case, the ball $\overline{\mathcal{B}}_{\sigma}(\bar{x})$ in (12a) can be replaced by $\left\{x_{\varepsilon}\right\}$.

To construct an admissible solution to $\mathcal{O C P}_{T}\left(x_{\delta}\right)$ from the optimal solution to $\mathcal{O C P}_{T}\left(x_{0}\right)$, we define the intervals

$$
\begin{aligned}
& \mathcal{I}_{1}:=\left[0, \tau_{2}(T, \varepsilon)-\delta\right), \\
& \mathcal{I}_{2}:=\left[\tau_{2}(T, \varepsilon)-\delta, \tau_{2}(T, \varepsilon)\right) \\
& \mathcal{I}_{3}:=\left[\tau_{2}(T, \varepsilon), T\right]
\end{aligned}
$$

and consider the following input trajectory

$$
u(\tau, \delta)=\left\{\begin{array}{ll}
u^{\star}\left(\tau+\delta, x_{0}\right) & \tau \in \mathcal{I}_{1} \\
u_{\varepsilon}\left(\tau-\tau_{2}(T, \varepsilon)+\delta, x_{\varepsilon}\right) & \tau \in \mathcal{I}_{2} \\
u^{\star}\left(\tau, x_{0}\right) & \tau \in \mathcal{I}_{3}
\end{array},\right.
$$

where $x_{\varepsilon}:=x\left(\tau_{2}(T, \varepsilon)-\delta, x_{0}, u^{\star}\left(\cdot, x_{0}\right)\right)$ and $u_{\varepsilon}\left(\cdot, x_{\varepsilon}\right)$ satisfies (12).

Lemma 3 (Recursive feasibility): Let Assumptions 2-4 hold. Then, there exists an horizon length $T>0$ such that $u(\cdot, \delta)$ from $(15)$ is an admissible input to $\mathcal{O C P}_{T}\left(x_{\delta}\right)$.

Proof: From Assumption 3, for all sufficiently large $T$, the optimal solutions to $\mathcal{O C P}_{T}\left(x_{0}\right)$ have a nonpathological turnpike property, i.e., for any $\varepsilon \in(0, \hat{\varepsilon}]$ and all $\tau \in\left[\tau_{1}\left(x_{0}, \varepsilon\right), \tau_{2}(T, \varepsilon)\right]$, the optimal solutions satisfy $x^{\star}\left(\tau, x_{0}, u^{\star}\left(\cdot, x_{0}\right)\right) \in \mathcal{B}_{\varepsilon}(\bar{x})$. We proceed by investigating the properties of $x\left(\cdot, x_{\delta}, u(\cdot, \delta)\right)$ for the intervals $\mathcal{I}_{i}, i \in$ $\{1,2,3\}$ separately.

Interval $\mathcal{I}_{1}$ : Consider $x\left(\cdot, x_{\delta}, u(\cdot, \delta)\right)$. The construction of $u(\cdot, \delta)$ implies that, for all $\tau \in \mathcal{I}_{1}$, we have $u(\tau, \delta)=$ $u^{\star}\left(\tau+\delta, x_{0}\right)$, and thus $x\left(\tau, x_{\delta}, u(\cdot, \delta)\right) \in \mathcal{X}$.

Interval $\mathcal{I}_{2}$ : The construction of $u(\cdot, \delta)$ implies

$$
x_{\varepsilon}=x\left(\tau_{2}(T, \varepsilon)-\delta, x_{\delta}, u^{\star}\left(\cdot, x_{0}\right)\right) .
$$

From Assumption 3, we have $x_{\varepsilon} \in \mathcal{B}_{\varepsilon}(\bar{x})$, and by Assumption 4 , (12) holds. In other words, for $\tau \in \mathcal{I}_{2}$, the $u_{\varepsilon}$-part of (15) is such that $x\left(\tau, x_{\delta}, u(\cdot, \delta)\right) \in \overline{\mathcal{B}}_{\sigma}(\bar{x}) \subset \mathcal{X}$.

Interval $\mathcal{I}_{3}$ : Again, we use the fact that the $u_{\varepsilon}$-part of $u(\cdot, \delta))$ implies $x\left(\tau_{2}(T, \varepsilon), x_{\delta}, u(\cdot, \delta)\right)=x\left(\tau_{2}(T, \varepsilon)-\right.$ $\left.\delta, x_{0}, u^{\star}\left(\cdot, x_{0}\right)\right)$. Hence, application of $u(\tau, \delta)=u^{\star}\left(\tau, x_{0}\right)$ for all $\tau \in \mathcal{I}_{3}$ leads to $x\left(\tau, x_{\delta}, u(\cdot, \delta)\right) \in \mathcal{X}$.

\section{Decrease of $J^{\star}\left(x_{\delta}\right)-J^{\star}\left(x_{0}\right)$}

In the following, we derive an upper bound on $J^{\star}\left(x_{\delta}\right)-$ $J^{\star}\left(x_{0}\right)$. Later, this bound will be essential in showing convergence of the NMPC scheme to a neighborhood of $\bar{x}$. To this end, consider the closed ball $\overline{\mathcal{B}}_{\varrho}(\bar{x}) \subset \mathbb{R}^{n_{x}}$. The distance of any point $x$ to this ball is defined as

$$
\mathrm{d}\left(x, \overline{\mathcal{B}}_{\varrho}(\bar{x})\right):=\min _{\xi \in \overline{\mathcal{B}}_{\varrho}(\bar{x})}\|\xi-x\| .
$$

Lemma 4 (Cost decrease): Let Assumptions 2-4 hold for $x_{0} \in \mathcal{X}_{0}$, let $I\left(x_{p}\left(t_{k}\right)\right)=S\left(x_{p}\left(t_{k}\right)\right)$ with $S$ from (9), and let 
$T$ be sufficiently large. Then, there exists $\varrho_{\text {min }}>0$ such that if, for all $\tau \in[0, \delta], x^{\star}\left(\tau, x_{0}\right) \notin \overline{\mathcal{B}}_{\varrho}(\bar{x})$, with $\varrho \geq \varrho_{\text {min }}>0$,

$$
J^{\star}\left(x_{\delta}\right)-J^{\star}\left(x_{0}\right) \leq-\int_{0}^{\delta} \mathrm{d}\left(x^{\star}\left(\tau, x_{0}\right), \overline{\mathcal{B}}_{\varrho}(\bar{x})\right) d \tau
$$

holds.

Proof: First, recall that Assumptions 2-4 lead to Lemma 3. It follows that $u(\cdot, \delta)$ given by $(15)$ is admissible in $\mathcal{O C P} \mathcal{P}_{T}\left(x_{\delta}\right)$ and, by optimality,

$$
J^{\star}\left(x_{\delta}\right)-J^{\star}\left(x_{0}\right) \leq \Delta J
$$

with $\Delta J:=J\left(x_{\delta}, u(\cdot, \delta)\right)-J^{\star}\left(x_{0}\right)$.

We use the properties of $u(\cdot, \delta)$ and investigate the RHS of (18) separately for $\mathcal{I}_{1}$ and $\mathcal{I}_{3}$. Equation (15) implies that

$$
x\left(\tau, x_{\delta}, u(\cdot, \delta)\right)=x\left(\tau+\delta, x_{0}, u^{\star}\left(\cdot, x_{0}\right)\right) \text { for } \tau \in \mathcal{I}_{1} .
$$

Hence, we obtain the identity

$$
\begin{array}{r}
\int_{0}^{\tau_{2}-\delta} F\left(z\left(\tau, x_{\delta}, u(\cdot, \delta)\right)\right) d \tau-\int_{0}^{\tau_{2}} F\left(z^{\star}\left(\tau, x_{0}\right)\right) d \tau \\
=-\int_{0}^{\delta} F\left(z^{\star}\left(\tau, x_{0}\right)\right) d \tau
\end{array}
$$

Similarly, it follows from (12) and (15) that

$$
x\left(\tau, x_{\delta}, u(\cdot, \delta)\right)=x\left(\tau, x_{0}, u^{\star}\left(\cdot, x_{0}\right)\right) \quad \text { for } \tau \in \mathcal{I}_{3},
$$

and thus

$$
\int_{\mathcal{I}_{3}} F\left(z\left(\tau, x_{\delta}, u(\cdot, \delta)\right)\right) d \tau-\int_{\mathcal{I}_{3}} F\left(z^{\star}\left(\tau, x_{0}\right)\right) d \tau=0 .
$$

We obtain from (19)

$$
\begin{aligned}
\Delta J \leq S\left(x_{\delta}\right)-S\left(x_{0}\right) & -\int_{0}^{\delta} F\left(z^{\star}\left(\tau, x_{0}\right)\right) d \tau \\
& +\int_{\mathcal{I}_{2}} F\left(z\left(\tau, x_{\varepsilon}, u_{\varepsilon}\left(\cdot, x_{\varepsilon}\right)\right) d \tau .\right.
\end{aligned}
$$

The dissipation inequality (9) can be written as

$$
\begin{aligned}
-\int_{0}^{\delta} F\left(z^{\star}\left(\tau, x_{0}\right)\right) d \tau \leq S\left(x_{0}\right)-S\left(x_{\delta}\right) \\
-\int_{0}^{\delta} \alpha\left(\left\|z^{\star}\left(\tau, x_{0}\right)-\bar{z}\right\|\right)+F(\bar{z}) d \tau .
\end{aligned}
$$

Using the last inequality, (20) can be written as

$$
\begin{aligned}
\Delta J \leq-\int_{0}^{\delta} \alpha\left(\| z^{\star}\left(\tau, x_{0}\right)\right. & -\bar{z} \|)+F(\bar{z}) d \tau \\
& +\int_{\mathcal{I}_{2}} F\left(z\left(\tau, x_{\varepsilon}, u_{\varepsilon}\left(\cdot, x_{\varepsilon}\right)\right) d \tau .\right.
\end{aligned}
$$

Recall that $\mathcal{I}_{2}$ is of length $\delta$, which gives

$$
\begin{aligned}
\Delta J \leq-\int_{0}^{\delta} & \alpha\left(\left\|z^{\star}\left(\tau, x_{0}\right)-\bar{z}\right\|\right) d \tau \\
& +\int_{\mathcal{I}_{2}} F\left(z\left(\tau, x_{\varepsilon}, u_{\varepsilon}\left(\cdot, x_{\varepsilon}\right)\right)-F(\bar{z}) d \tau .\right.
\end{aligned}
$$

Now, we apply the bound given in Lemma 6 in the Appendix to the last integral in (22), which gives

$$
\Delta J \leq-\int_{0}^{\delta} \alpha\left(\left\|z^{\star}\left(\tau, x_{0}\right)-\bar{z}\right\|\right)-L_{F}(\varepsilon+\eta) e^{L_{f} \delta} d \tau .
$$

Since $z=(x, u)^{T}$, we have $\|z\| \geq\|x\|$, and therefore

$$
\Delta J \leq-\int_{0}^{\delta} \alpha\left(\left\|x^{\star}\left(\tau, x_{0}\right)-\bar{x}\right\|\right)-L_{F}(\varepsilon+\eta) e^{L_{f} \delta} d \tau
$$

Note that, for any closed ball $\overline{\mathcal{B}}_{\varrho}(\bar{x})$, the identity

$$
\mathrm{d}\left(x, \overline{\mathcal{B}}_{\varrho}(\bar{x})\right)=\|x-\bar{x}\|-\varrho, \quad \forall x \notin \overline{\mathcal{B}}_{\varrho}(\bar{x})
$$

holds. Now, picking $\varrho_{\text {min }}=\alpha^{-1}\left(L_{F}(\varepsilon+\eta) e^{L_{f} \delta}\right)$ and recalling that, for all $\tau \in[0, \delta]$, we have assumed $x\left(\tau, x_{0}, u^{\star}\left(\cdot, x_{0}\right)\right) \notin \overline{\mathcal{B}}_{\varrho}(\bar{x})$, assertion (17) follows from (23) and (18).

\section{CONVERGENCE OF NMPC VIA TURnPIKES}

Lemmas 2-3 indicate that (non-pathological) approximate turnpike properties allow easy construction of admissible solutions to receding-horizon sequences of $\mathcal{O C P}_{T}\left(x_{p}\left(t_{k}\right)\right)$ as they arise in NMPC. Additionally, Lemma 4 establishes a cost decrease property. Hence, we use approximate turnpike properties to establish (i) convergence to a turnpike neighborhood and (ii) recursive feasibility of sampled-data NMPC.

Consider the plant (1) controlled by the NMPC scheme based on $\mathcal{O C P}_{T}\left(x_{p}\left(t_{k}\right)\right)$ that generates the input (5).

Proposition 2 (Convergence to a turnpike neighborhood): Let Assumptions 1-4 hold, and let the penalty on the initial condition be $I\left(x_{p}\left(t_{k}\right)\right)=S\left(x_{p}\left(t_{k}\right)\right)$, with $S$ from (9). Then, there exists an horizon length $T$ and a sampling time $\delta$ such that the following statements hold:

(i) If $\mathcal{O C P}_{T}\left(x_{p}\left(t_{k}\right)\right)$ is initially feasible, it remains feasible at all sampling instants $t_{k}>0$.

(ii) For all $x_{0} \in \mathcal{X}_{0}$, the closed-loop trajectories satisfy

$$
\lim _{t \rightarrow \infty} \mathrm{d}\left(x_{p}\left(t, x_{0}, u_{p}(\cdot)\right), \overline{\mathcal{B}}_{\hat{\varrho}}(\bar{x})\right)=0
$$

with

$$
\hat{\varrho}=\alpha^{-1}\left(L_{F}(\varepsilon+\eta) e^{L_{f} \delta}\right)+\gamma(\delta),
$$

and $\gamma(\delta)=(\varrho+\eta)\left(e^{L_{f} \delta}-1\right)$.

Proof: Recursive feasibility of $\mathcal{O C P}_{T}\left(x_{p}\left(t_{k}\right)\right)$ follows from Lemmas 2 and 3. The remainder of our proof is similar to a convergence proof for set-based sampled-data NMPC presented in [8, 9]. It is based on a set-based extension of Barbalat's Lemma [8, Lemma 4.1, p. 50]. Due to space limitations, we only discuss the main steps: First, we introduce a suitable value function, which does not need to be continuous in $x_{p}$. Second, we analyze the behavior of $V\left(x_{p}\right)$ for $t \in\left[t_{k}, t_{k}+\delta\right)$, i.e., between two instants $t_{k}$ and $t_{k+1}$. Finally, we compare $V\left(x_{p}\left(t_{k}\right)\right)$ and $V\left(x_{p}\left(t_{k+1}\right)\right)$, i.e., we investigate the behavior of the value function from sampling instant $t_{k}$ to sampling instant $t_{k+1}$. 
In order to show convergence to $\overline{\mathcal{B}}_{\hat{\varrho}}(\bar{x})$, we consider the value function

$$
V\left(x_{p}\right)=\left\{\begin{array}{ll}
J^{\star}\left(x_{p}\right) & x_{p} \notin \overline{\mathcal{B}}_{\varrho}(\bar{x}) \\
0 & x_{p} \in \overline{\mathcal{B}}_{\varrho}(\bar{x})
\end{array},\right.
$$

which, since $S: \mathcal{X} \rightarrow \mathbb{R}_{0}^{+}$and $F: \mathcal{Z} \rightarrow \mathbb{R}_{0}^{+}$, is positive definite with respect to $\overline{\mathcal{B}}_{\varrho}(\bar{x}) \subset \overline{\mathcal{B}}_{\hat{\varrho}}(\bar{x})$. Note that feasibility at $t_{k}=0$ implies $V\left(x_{0}\right)<\infty$.

Decrease of $V\left(x_{p}(t)\right)$ between $t_{k}$ and $t_{k+1}$ : Consider $t \in$ $\left[t_{k}, t_{k}+\delta\right)$ and $x_{p}\left(t_{k}\right) \notin \overline{\mathcal{B}}_{\varrho}(\bar{x})$. Since the model is identical to the plant (Assumption 1), we have

$$
V\left(x_{p}(t)\right)=J^{\star}\left(x_{p}\left(t_{k}\right)\right)-\int_{t_{k}}^{t} F\left(z^{\star}\left(\tau, x_{p}\left(t_{k}\right)\right)\right) d \tau,
$$

i.e., the $V$ decreases between $t_{k}$ and $t_{k+1}$.

Decrease of $V\left(x_{p}(t)\right)$ from $t_{k}$ to $t_{k+1}$ : Since $\hat{\varrho} \geq \varrho$, and provided $x_{p}(t) \notin \overline{\mathcal{B}}_{\varrho}(\bar{x})$ for all $t \in\left[t_{k}, t_{k}+\delta\right)$, we can infer from Lemma 4 that $V\left(x_{p}\left(t_{k+1}\right)\right)-V\left(x_{p}\left(t_{k}\right)\right)<0$, i.e., $V$ decreases. Clearly, $V$ also decreases whenever $x_{p}\left(t_{k}\right) \notin$ $\overline{\mathcal{B}}_{\varrho}(\bar{x})$ and $x_{p}\left(t_{k+1}\right) \in \overline{\mathcal{B}}_{\varrho}(\bar{x})$. Combining these observations yields

$$
V\left(x_{p}\left(t_{k+1}\right)\right)-V\left(x_{p}\left(t_{k}\right)\right)<0, \quad \forall x_{p}\left(t_{k}\right) \notin \overline{\mathcal{B}}_{\varrho}(\bar{x}) .
$$

It remains to analyze the case $x_{p}\left(t_{k}\right) \in \overline{\mathcal{B}}_{\varrho}(\bar{x})$. For the moment, assume that the NMPC feedback renders $\overline{\mathcal{B}}_{\varrho}(\bar{x})$ positively invariant, i.e., it ensures that $x_{p}\left(t_{k+1}\right) \in \overline{\mathcal{B}}_{\varrho}(\bar{x})$ whenever $x_{p}\left(t_{k}\right) \in \overline{\mathcal{B}}_{\varrho}(\bar{x})$. Hence, a set-based extension to Barbalat's Lemma, such as the ones stated in $[8,9]$, can be used to establish convergence to $\overline{\mathcal{B}}_{\varrho}(\bar{x})$.

Next, assume the contrary, i.e., the plant driven by the NMPC input leaves $\overline{\mathcal{B}}_{\varrho}(\bar{x})$. To tackle this case, we consider the bound given in Lemma 5 in the Appendix. By (31), there exists a continuous function $\gamma(\delta)$ such that, for all $x_{p}\left(t_{k}\right) \in \overline{\mathcal{B}}_{\varrho}(\bar{x})$, it holds that $x_{p}\left(t_{k+1}\right) \in \overline{\mathcal{B}}_{\hat{\varrho}}(\bar{x})$ with $\hat{\varrho}=\varrho+\gamma(\delta), \gamma(0)=0$. In other words, due to the absence of plant-model mismatch, the plant state has to stay within a neighborhood of $\overline{\mathcal{B}}_{\varrho}(\bar{x})$. We may conclude from (26) and (27) that, for all $x_{p}\left(t_{k}\right) \in \overline{\mathcal{B}}_{\hat{\varrho}}(\bar{x}) \backslash \overline{\mathcal{B}}_{\varrho}(\bar{x})$, the plant state moves towards $\overline{\mathcal{B}}_{\varrho}(\bar{x})$. This holds from $t_{k}$ to $t_{k+1}$ and inbetween the instants $t_{k}$ and $t_{k+1}$, i.e., for $t \in\left[t_{k}, t_{k}+\delta\right)$. Combining the previous statements, we obtain via Lemma 4.1 in [8, p. 50], or via [9, Lem. 4.5], that (24) holds.

Remark 2 (Quantifying the size of $\overline{\mathcal{B}}_{\hat{\varrho}}(\bar{x})$ ): At this point, it is fair to ask for a quantification of the size of the set $\overline{\mathcal{B}}_{\hat{\varrho}}(\bar{x})$ to which the NMPC-controlled plant converges. Recall that $\hat{\varrho}=\hat{\varrho}(\varepsilon, \eta, \delta)=\alpha^{-1}\left(L_{F}(\varepsilon+\eta) e^{L_{f} \delta}\right)+\gamma(\delta)$ describes the size of $\overline{\mathcal{B}}_{\hat{\varrho}}(\bar{x})$. The first term $\alpha^{-1}\left(L_{F}(\varepsilon+\eta) e^{L_{f} \delta}\right)$ can be understood as a measure of the size of the deviation from the turnpike that happens upon application of the suboptimal admissible input (15). The second term $\gamma(\delta)$ accounts for the fact that the decrease (17) holds only outside of $\overline{\mathcal{B}}_{\varrho}(\bar{x})$.

Clearly, the size of $\overline{\mathcal{B}}_{\hat{\varrho}}(\bar{x})$ shrinks to 0 as $\|(\varepsilon, \eta, \delta)\| \rightarrow$ 0 . Recall that, in the view of Lemma 1 , we may choose $\eta>0, \delta>0$ arbitrarily small, and still we have small-time controllability on some $\varepsilon$-neighborhood of the turnpike $\bar{x}$. Furthermore, Assumption 3 ensures that, for some horizon
$T$, the optimal solutions are within any arbitrarily small $\varepsilon$ neighborhood of $\bar{x}$ for some part of $[0, T]$. Combining both observations we obtain that, by enlarging $T$ and shrinking $\delta$, $\hat{\varrho}(\varepsilon, \eta, \delta)$ can be made arbitrarily small.

Finally, it is worth mentioning that the penalty on the initial condition $I$ in (2) can be dropped, without loss of convergence properties, i.e., explicit knowledge of the storage function $S$ is not required.

Corollary 1 (Convergence with $\left.I\left(x_{p}\left(t_{k}\right)\right)=0\right)$ :

Let Assumptions 1-4 hold, and the penalty on the initial condition be $I\left(x_{p}\left(t_{k}\right)\right)=0$. Then, there exist $T>0$ and $\delta>0$ such that Statements (i)-(ii) in Proposition 2 hold. $\square$

Proof: Note that, for all $t_{k}$, the initial condition $x_{p}\left(t_{k}\right)$ is not a decision variable of $\mathcal{O C P}_{T}\left(x_{p}\left(t_{k}\right)\right)$. It is merely a translation of $J^{\star}\left(x_{p}\left(t_{k}\right)\right)$. Hence, any optimal solution to $\mathcal{O C P} \mathcal{P}_{T}\left(x_{p}\left(t_{k}\right)\right)$, obtained with $I\left(x_{p}\left(t_{k}\right)\right)=0$, is also an optimal solution to $\mathcal{O C P}_{T}\left(x_{p}\left(t_{k}\right)\right)$ with $I\left(x_{p}\left(t_{k}\right)\right)=S\left(x_{p}\left(t_{k}\right)\right)$. Therefore, setting $I\left(x_{p}\left(t_{k}\right)\right)=0$, gives the same closed-loop trajectories as in Proposition 2. Hence, the conclusions of Proposition 2 also hold for $I\left(x_{p}\left(t_{k}\right)\right)=0$.

\section{EXAMPLE - ECONOMIC NMPC FOR A CSTR}

We illustrate our results with the example of a continuous stirred-tank reactor [13]. A model of the reactor for the concentrations of species $A$ and $B$ and the reactor temperature $\vartheta$ reads

$$
\begin{aligned}
\dot{c}_{A} & =-r_{1}\left(c_{A}, \vartheta\right)-2 r_{3}\left(c_{A}, \vartheta\right)+\left(c_{i n}-c_{A}\right) v \\
\dot{c}_{B} & =r_{1}\left(c_{A}, \vartheta\right)-r_{2}\left(c_{B}, \vartheta\right)-c_{B} v \\
\dot{\vartheta} & =h\left(c_{A}, c_{B}, \vartheta\right)+\alpha(u-\vartheta)+\left(\vartheta_{i n}-\vartheta\right) v,
\end{aligned}
$$

where the input $u$ is the temperature in the cooling jacket. The numerical values of the model parameters and the terms $r_{i}, h$ can be found in [13]. The state and the input are subject to the constraints

$$
\begin{array}{llll}
c_{A} & \in[0,6] \mathrm{mol} / \mathrm{l} & c_{B} & \in[0,4] \mathrm{mol} / \mathrm{l} \\
\vartheta & \in[70,150]^{\circ} \mathrm{C} & u & \in[0,200]^{\circ} \mathrm{C} .
\end{array}
$$

For maximizing the average production rate of $c_{B}$, we specify the cost function as $F\left(c_{B}\right)=\beta v c_{B}, \quad \beta, v>0$. The OCP

$$
\underset{u(\cdot)}{\operatorname{maximize}} \int_{0}^{T} \beta v c_{B}(\tau) d \tau \quad \text { s.t. (28) and (29) }
$$

has an approximate turnpike $4^{4}$ at $\left(\bar{c}_{A}, \bar{c}_{B}, \bar{\vartheta}, \bar{u}\right)=$ $(2.175,1.105,128.53,142.76)$. Furthermore, the linearization of (28) at this steady state is controllable.

We compute a solution to OCP (30) with $T=0.25 h$ and $c_{A}(0)=1.0 \mathrm{~mol} / l, c_{B}(0)=0.78 \mathrm{~mol} / l, T(0)=142{ }^{\circ} \mathrm{C}$ using a piecewise-constant input parametrization via direct multiple shooting. These open-loop optimal results are depicted by continuous grey lines in Figure 2. As one can see, the open-loop optimal solutions have an input-state turnpike

\footnotetext{
${ }^{4}$ It is easy to check that, for constant $v=35 h^{-1}$, the approximate turnpike property presented in [7] holds. Therein, using a fourth-order polynomial approximation of $r_{i}, h$, it is also shown that the turnpike takes place at the globally optimal steady state.
} 

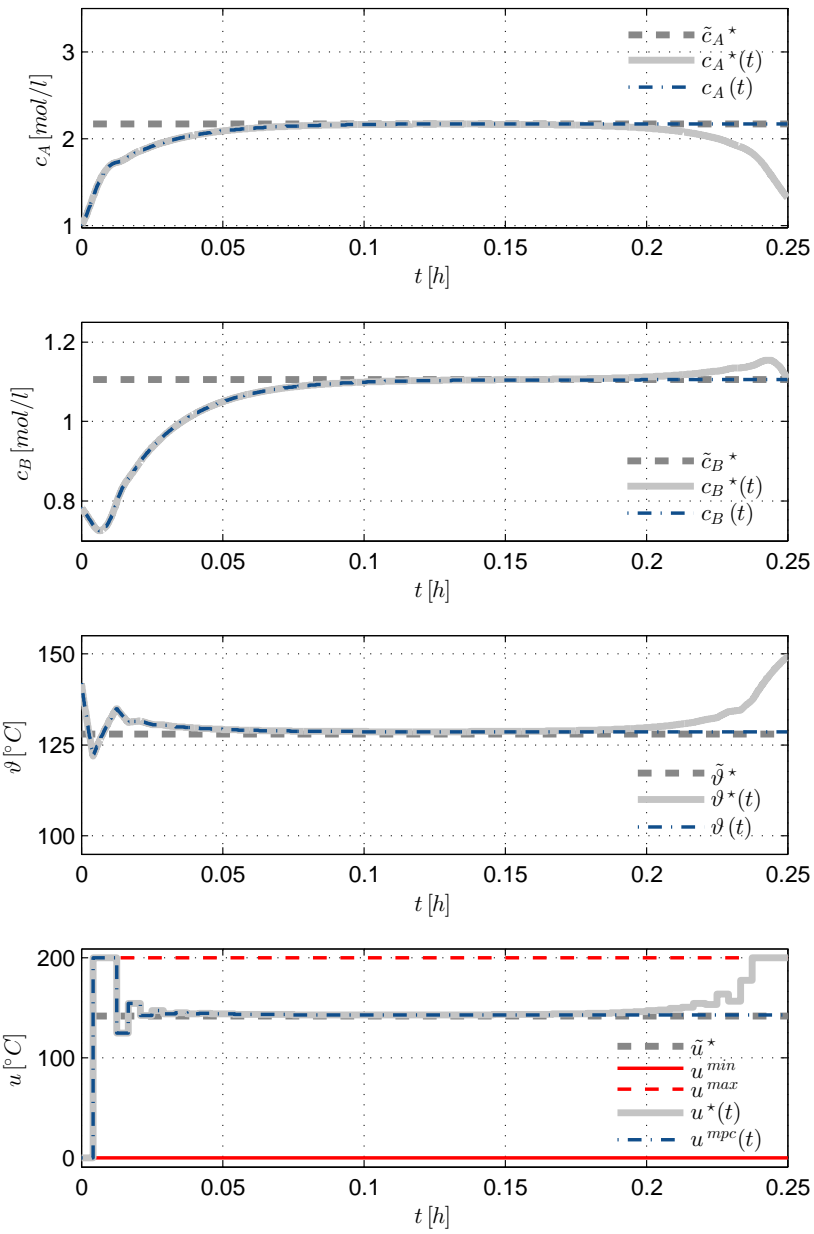

Fig. 2. Economic NMPC for a continuous reactor with turnpike. The turnpike corresponds to the optimal steady state.

at $\left(\bar{c}_{A}, \bar{c}_{B}, \bar{\vartheta}, \bar{u}\right)$. This turnpike, i.e., the optimal steady-state pair, is illustrated by grey dashed lines. Next, we simulate an NMPC scheme based on receding-horizon solutions to (30). We use the prediction horizon $T=0.25 h$ and the sampling time $\delta=0.004 h$. The results are depicted by dash-dot lines in Figure 2. As one can see, and as predicted by our results, the closed-loop solutions converge rapidly to the turnpike.

\section{CONCLUSIONS}

This paper has presented novel sufficient convergence conditions for sampled-data (economic) NMPC schemes with input and state constraints. The proposed conditions, which are based on an approximate turnpike property, do neither require terminal penalties nor terminal constraints. A key ingredient in our developments is the use of a storage function as a penalty on the initial condition in the NMPC scheme. We also investigated conditions guaranteeing recursive feasibility of the optimization.

\section{APPENDIX}

Lemma 5 (Bound on solutions leaving $\overline{\mathcal{B}}_{\varrho}(\bar{x})$ ):

Let Assumption 2 hold, and $x(\delta):=x\left(\delta, x_{0}, u(\cdot)\right)$ be the state reached from any $x_{0} \in \overline{\mathcal{B}}_{\varrho}(\bar{x})$ at time $\delta$ upon application of an admissible input $u(\cdot) \in \mathcal{L}\left([0, \delta], \mathcal{B}_{\eta}(\bar{u})\right)$. Furthermore, let $L_{f}$ be a Lipschitz constant of (1) with respect to $(x, u)$. Then, for all $\delta \geq 0$,

$$
\|x(\delta)-\bar{x}\| \leq \varrho+\gamma(\delta),
$$

with $\gamma(\delta)=(\varrho+\eta)\left(e^{L_{f} \delta}-1\right)$.

Proof: The proof follows from Lipschitz continuity of $f$ and Gronwall's inequality.

Lemma 6: Let Assumptions 2 and 4 hold, and let $L_{F}$ be a Lipschitz constant of $F$ with respect to $(x, u)$. Then, for any $x_{\varepsilon} \in \mathcal{B}_{\varepsilon}(\bar{x})$ and all $\tau \in[0, \delta]$, the admissible pair $z_{\varepsilon}(\cdot)=$ $z_{\varepsilon}\left(\cdot, x_{\varepsilon}, u_{\varepsilon}(\cdot)\right)$ driven by $u_{\varepsilon}(\cdot) \in \mathcal{L}\left([0, T], \mathcal{B}_{\eta}(\bar{u})\right)$ from (12) satisfies $F\left(z_{\varepsilon}(\tau)\right)-F(\bar{z}) \leq L_{F}(\eta+\varepsilon) e^{L_{f} \delta}$.

Proof: The proof follows directly from Lipschitz continuity of $F$ and Lemma 5.

\section{BIBLIOGRAPHY}

[1] D.A. Carlson, A. Haurie, and A. Leizarowitz. Infinite Horizon Optimal Control: Deterministic and Stochastic Systems. Springer Verlag, 1991.

[2] T. Damm, L. Grüne, M. Stieler, and K. Worthmann. "An exponential turnpike theorem for dissipative optimal control problems". In: SIAM Journal on Control and Optimization 52:3 (2014), pp. 1935-1957.

[3] M. Diehl, R. Amrit, and J.B. Rawlings. "A Lyapunov function for economic optimizing model predictive control". In: IEEE Trans. Automat. Contr. 56:3 (2011), pp. 703-707.

[4] R. Dorfman, P.A. Samuelson, and R.M. Solow. Linear Programming and Economic Analysis. McGraw-Hill, New York, 1958.

[5] M. Ellis, H. Durand, and P.D. Christofides. "A tutorial review of economic model predictive control methods". In: Journal of Process Control 24:8 (2014), pp. 1156-1178.

[6] T. Faulwasser and D. Bonvin. "On the design of economic NMPC based on an exact turnpike property". In: 9th IFAC International Symposium on Advanced Control of Chemical Processes (ADCHEM). Whistler, BC, Canada, 2015.

[7] T. Faulwasser, M. Korda, C.N. Jones, and D. Bonvin. "Turnpike and dissipativity properties in dynamic real-time optimization and economic MPC". In: Proc. of the 53rd IEEE Conference on Decision and Control (CDC). Los Angeles, California, USA, 2014, pp. 2734-2739.

[8] R. Findeisen. Nonlinear Model Predictive Control: A Sampled-Data Feedback Perspective. Fortschr.-Ber. VDI Reihe 8 Nr. 1087. VDI Verlag, Düsseldorf, 2006.

[9] F. Fontes and L. Magni. "Min-max model predictive control of nonlinear systems using discontinuous feedbacks". In: IEEE Trans. Aut. Contr. 48:10 (2003), pp. 1750-1755.

[10] L. Grüne. "Economic receding horizon control without terminal constraints". In: Automatica 49:3 (2013), pp. 725-734. ISSN: 0005-1098.

[11] L. Grüne and M. Stieler. "A Lyapunov function for economic MPC without terminal conditions". In: Decision and Control (CDC), 2014 IEEE 53rd Annual Conference on. 2014, pp. 2740-2745.

[12] L.W. McKenzie. "Turnpike theory". In: Econometrica: Journal of the Econometric Society 44:5 (1976), pp. 841-865.

[13] R. Rothfuß, J. Rudolph, and M. Zeitz. "Flatness based control of a nonlinear chemical reactor model". In: Automatica 32 (1996), pp. 1433-1439.

[14] S. Sastry. Nonlinear Systems: Analysis, Stability, and Control. Vol. 10. Springer New York, 1999. 